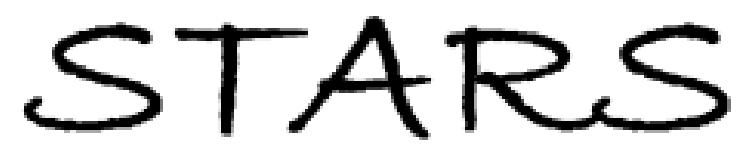

University of Central Florida

STARS

$1-1-2008$

\title{
Dynamical scaling exponents for polymer translocation through a nanopore
}

Kaifu Luo

Santtu T. T. Ollila

Ilkka Huopaniemi

Tapio Ala-Nissila

Pawel Pomorski

See next page for additional authors

Find similar works at: https://stars.library.ucf.edu/facultybib2000

University of Central Florida Libraries http://library.ucf.edu

This Article is brought to you for free and open access by the Faculty Bibliography at STARS. It has been accepted for inclusion in Faculty Bibliography 2000s by an authorized administrator of STARS. For more information, please contactSTARS@ucf.edu.

\section{Recommended Citation}

Luo, Kaifu; Ollila, Santtu T. T.; Huopaniemi, Ilkka; Ala-Nissila, Tapio; Pomorski, Pawel; Karttunen, Mikko; Ying, See-Chen; and Bhattacharya, Aniket, "Dynamical scaling exponents for polymer translocation through a nanopore" (2008). Faculty Bibliography 2000s. 661.

https://stars.library.ucf.edu/facultybib2000/661

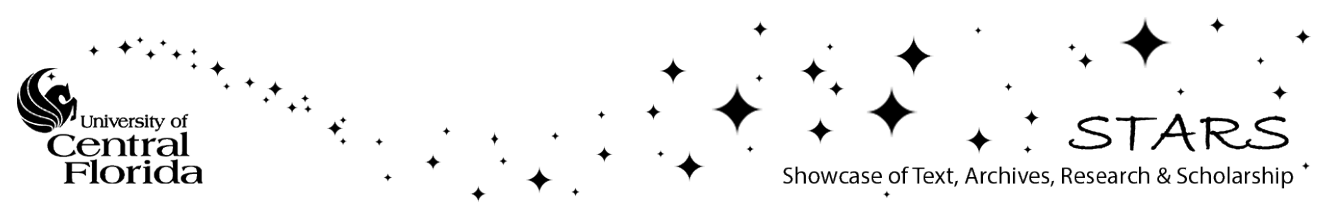




\section{Authors}

Kaifu Luo, Santtu T. T. Ollila, Ilkka Huopaniemi, Tapio Ala-Nissila, Pawel Pomorski, Mikko Karttunen, SeeChen Ying, and Aniket Bhattacharya 


\title{
Dynamical scaling exponents for polymer translocation through a nanopore
}

\author{
Kaifu Luo, ${ }^{1}$ Santtu T. T. Ollila, ${ }^{1}$ Ilkka Huopaniemi, ${ }^{1}$ Tapio Ala-Nissila, ${ }^{1,2}$ Pawel Pomorski, ${ }^{3}$ Mikko Karttunen, ${ }^{3}$ \\ See-Chen Ying, ${ }^{2}$ and Aniket Bhattacharya ${ }^{4}$ \\ ${ }^{1}$ Department of Applied Physics, Helsinki University of Technology, P.O. Box 1100, FIN-02015 TKK, Espoo, Finland \\ ${ }^{2}$ Department of Physics, Box 1843, Brown University, Providence, Rhode Island 02912-1843, USA \\ ${ }^{3}$ Department of Applied Mathematics, The University of Western Ontario, London, Ontario N6A 5B7, Canada \\ ${ }^{4}$ Department of Physics, University of Central Florida, Orlando, Florida 32816-2385, USA \\ (Received 27 May 2008; revised manuscript received 13 August 2008; published 3 November 2008)
}

\begin{abstract}
We determine the scaling exponents of polymer translocation (PT) through a nanopore by extensive computer simulations of various microscopic models for chain lengths extending up to $N=800$ in some cases. We focus on the scaling of the average PT time $\tau \sim N^{\alpha}$ and the mean-square change of the PT coordinate, $\left\langle s^{2}(t)\right\rangle \sim t^{\beta}$. We find $\alpha=1+2 \nu$ and $\beta=2 / \alpha$ for unbiased PT in two dimensions (2D) and three dimensions (3D). The relation $\alpha \beta=2$ holds for driven PT in $2 \mathrm{D}$, with a crossover from $\alpha \approx 2 \nu$ for short chains to $\alpha \approx 1+\nu$ for long chains. This crossover is, however, absent in 3D where $\alpha=1.42 \pm 0.01$ and $\alpha \beta \approx 2.2$ for $N \approx 40-800$.
\end{abstract}

DOI: 10.1103/PhysRevE.78.050901

PACS number(s): 87.15.A-, 82.35.Lr, 82.35.Pq

The transport of a polymer through a nanopore plays a crucial role in numerous biological processes, such as DNA and RNA translocation across nuclear pores, protein transport through membrane channels, and virus injection. Due to various potential technological applications, such as rapid DNA sequencing, gene therapy, and controlled drug delivery, polymer translocation has become a subject of intensive experimental [1,2] and theoretical studies [3-19,21-23].

Among the most fundamental quantities associated with translocation, the average translocation time $\tau$ as a function of the chain length $N$ is an important measure of the underlying dynamics. Standard equilibrium Kramers analysis [24] of diffusion through an entropic barrier yields $\tau \sim N^{2}$ for unbiased translocation and $\tau \sim N$ for driven translocation (assuming friction is independent of $N$ ) $[3,4]$. However, as Chuang et al. [5] noted, the quadratic scaling behavior for unbiased translocation cannot be correct for a self-avoiding polymer. The reason is that the translocation time is shorter than the Rouse equilibration time of a self-avoiding polymer, $\tau_{R} \sim N^{1+2 \nu}$, where the Flory exponent $\nu=0.588$ in three dimensions (3D) and $\nu=0.75$ in $2 \mathrm{D}$ [25], thus rendering the concept of equilibrium entropy and the ensuing entropic barrier inappropriate for translocation dynamics. Chuang et al. [5] performed numerical simulations with Rouse dynamics for a $2 \mathrm{D}$ lattice model to study the translocation for both phantom and self-avoiding polymers. They decoupled the translocation dynamics from the diffusion dynamics outside the pore by imposing the restriction that the first monomer, which is initially placed in the pore, be never allowed to cross back out of the pore. Their results show that for large $N, \tau \sim N^{1+2 \nu}$, which scales approximately in the same manner as the equilibration time, but with a much larger prefactor. This result was recently corroborated by extensive numerical simulations based on the fluctuating bond (FB) [6] and Langevin dynamics (LD) models with the bead-spring approach [7-10]. In Refs. [6,7] the translocation time $\tau$ was found to scale as $N^{2.50 \pm 0.01}$ in $2 \mathrm{D}$, which is in agreement with $\tau \sim N^{1+2 \nu}$.

For driven translocation, Kantor and Kardar [11] have demonstrated that the assumption of equilibrium in polymer dynamics by Sung and Park [3] and Muthukumar [4] breaks down more easily and provided a lower bound $\tau \sim N^{1+\nu}$ for the translocation time by comparison to the unimpeded motion of the polymer. Using FB [12] and LD [7,13] models, a crossover from $\tau \sim N^{1.46 \pm 0.01} \approx N^{2 \nu}$ for relatively short polymers to $\tau \sim N^{1.70 \pm 0.03} \approx N^{1+\nu}$ for longer chains was found in 2D.

Recently, however, alternate scaling scenarios have been presented [15-19,21], which contradict the above results. To resolve the apparent discrepancy, we have undertaken an extensive effort to determine $\tau$ as a function of $N, \tau \sim N^{\alpha}$, and the mean-square change of the translocation coordinate $\left\langle s^{2}(t)\right\rangle \sim t^{\beta}$ based on high-accuracy numerical simulations. The independent models employed here include the FB model with Monte Carlo (MC) dynamics [6,12] in 2D, standard LD of the bead-spring model of polymers $[7,8,13,14]$ in both $2 \mathrm{D}$ and 3D, and atomistic molecular dynamics (MD) simulations using the GROMACS [26] simulation engine in both $2 \mathrm{D}$ and $3 \mathrm{D}$.

In the 2D lattice FB model for MC simulation of a selfavoiding polymer [6,12], each segment excludes four nearest- and next-nearest-neighbor sites on a square lattice. The bond lengths $b_{l}$ vary in length, but do not cross each other. Dynamics is introduced by Metropolis moves of a single segment, with a probability of acceptance $\min \left(e^{-U / k_{B} T}, 1\right)$, where $U$ is the energy difference between the new and old states, $k_{B}$ the Boltzmann constant, and $T$ the absolute temperature. In a MC move, we randomly select a monomer and attempt to move it onto an adjacent lattice site (in a randomly selected direction). If the new position does not violate the excluded-volume or maximal bond-length restrictions, the move is accepted or rejected according to the Metropolis criterion. $N$ elementary moves define one MC time step.

In LD simulations $[8,13,14]$, the polymer chains are modeled as bead-spring chains of Lennard-Jones (LJ) particles with the finite-extension nonlinear elastic (FENE) potential. The excluded-volume interaction between monomers is modeled by a short-range $(\mathrm{SR})$ repulsive $\mathrm{LJ}$ potential with a cutoff of $2^{1 / 6} \sigma$, where $\sigma$ is the bead diameter. Between all monomer-wall particle pairs, there exists the same SR repulsive LJ interaction. In the LD simulations, each monomer is 


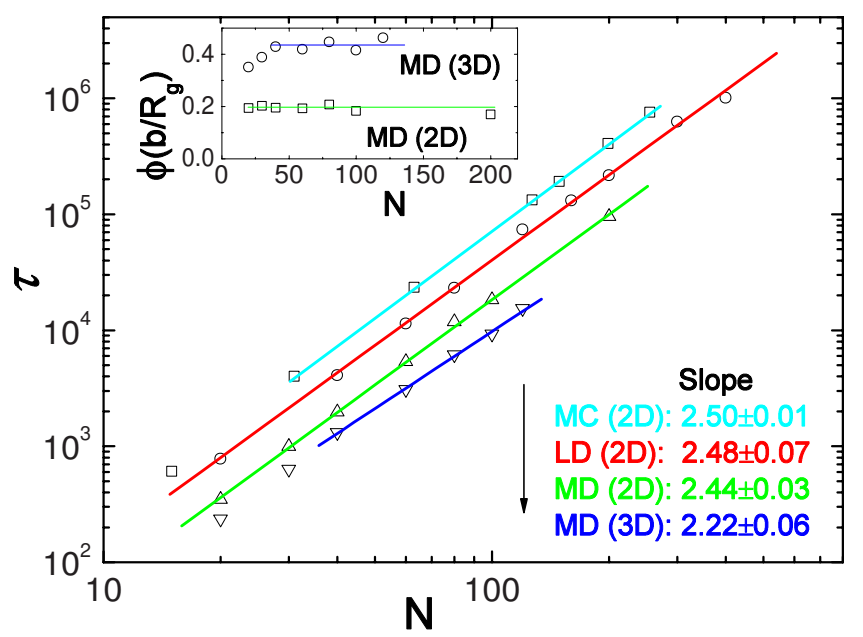

FIG. 1. (Color online) Scaling of the translocation time for unbiased case. Curves have been shifted for clarity. For MC and LD data in $2 \mathrm{D}$, the slopes are $2.50 \pm 0.01$ [6] and 2.48 \pm 0.07 [7], respectively. For MD data, the slopes are $2.44 \pm 0.03$ (2D) and $2.22 \pm 0.06(3 \mathrm{D})$, respectively. The solid lines indicate fitted data points.

subjected to conservative, frictional, and random forces, respectively.

GROMACS [26] is currently one of the most commonly used programs in soft-matter and biophysical simulations, and has also been used extensively by some of us in various problems (see, e.g., Ref. [27] and references therein). As in the MC and standard LD methods, the hydrodynamic effects are excluded from our GROMACS MD simulations. The GROMACS MD algorithm can be implemented with different thermostats. We have used both overdamped Brownian and Langevin dynamics thermostats. These yield the same exponents for $\tau$. The results shown here and labeled as MD are from the GROMACS algorithm with LD thermostats.

For unbiased translocation, the middle monomer is initially placed in the center of the pore. The polymer can escape the pore from either side in time defined as the translocation time $\tau$. We simulated the escape of chains of lengths varying from $N=15$ up to $N=255$ for the scaling of $\tau$ and averaged over 200 samples for MD simulations in both 2D and 3D and over 2000 samples for MC and LD simulations in $2 \mathrm{D}$ to minimize statistical errors.

Figure 1 shows $\tau \sim N^{\alpha}$ for different models. For MD simulations we find that $\alpha=2.44 \pm 0.03$ in $2 \mathrm{D}$ and $\alpha$ $=2.22 \pm 0.06$ in 3D, in complete agreement with $\alpha$ $=2.50 \pm 0.01$ from MC simulations in 2D [6] and $\alpha$ $=2.48 \pm 0.07$ from LD simulations in $2 \mathrm{D}$ [7]. All these results are consistent with the results from scaling arguments, $\tau \sim N^{1+2 \nu}[5-7]$, and also agree with the recent results by Wei et al. [9], where $\tau \sim N^{2.51 \pm 0.03}$ in 2D and $\tau \sim N^{2.2}$ in 3D based on LD simulations.

The scaling $\tau \sim N^{1+2 \nu}$ implies that $\tau$ scales in the same manner as the chain equilibration time $\tau_{R}$. Here, $\tau_{R}$ is the time it takes a polymer to move a distance equal to its radius of gyration $\tau_{R} \sim R_{g}^{2} / D, D=1 / N$ being the diffusion coefficient. Most recently, Slater and co-workers [22] used MD simulations with explicit solvent to study the impact of hy-

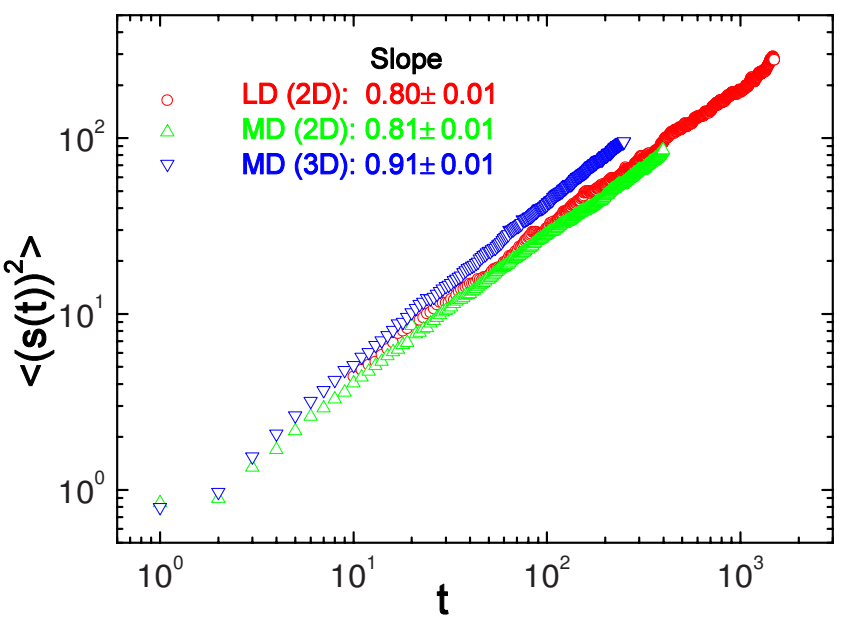

FIG. 2. (Color online) Scaling of the mean-square displacement of the translocation coordinate for unbiased case. For LD data in $2 \mathrm{D}$, the slope is $0.80 \pm 0.01$ for $10 \leqslant t \leqslant 350$. For GROMACS data, the slopes are $0.81 \pm 0.01(2 \mathrm{D})$ for $10 \leqslant t \leqslant 399$ and $0.91 \pm 0.01$ (3D) for $10 \leqslant t \leqslant 250$, respectively.

drodynamic interactions in $3 \mathrm{D}$. The results show that the scaling of the translocation time varies from $\tau \sim N^{1+2 \nu}$ to $\tau$ $\sim N^{3 \nu}$ with increasing pore size, which indicates that the hydrodynamic interaction is screened for small pore sizes. These results also support $\tau \sim R_{g}^{2} / D$ by taking into account $D \sim 1 / N$ and $D \sim 1 / N^{\nu}$ without and with hydrodynamic interactions, respectively. Using a similar argument, it was also predicted, and numerically confirmed, that $\tau \sim\left(R_{g}+L\right)^{2} / D$ $\sim N L^{2}$ for a long pore of length $L \gg R_{g}$, resulting from the fact that the center of mass of the polymer moves a distance of $L+R_{g} \approx L$ [6]. For a long pore $L \gg N$ we have $\tau \sim N L^{2}$ $\gg N^{3}$, which is longer than the reptation time of the chain, $\sim N^{3}$. In addition, for translocation under a pulling force $F$ acting on one end of the chain, $\tau \sim N^{2 \nu+1}$ is recovered for $F \rightarrow 0$ [8]. Altogether, these results further confirm the argument that $\tau$ scales in the same manner as $\tau_{R}$.

For the mean-square change of the translocation coordinate $s(t)$, we use chains of length $N=201$ for LD simulations in $2 \mathrm{D}$ and $N=100$ for MD simulations in both $2 \mathrm{D}$ and $3 \mathrm{D}$, and average over 2000 samples. As shown in Fig. 2, we observe subdiffusive behavior $\left\langle s^{2}(t)\right\rangle \sim t^{\beta}$, where $\beta$ $=0.80 \pm 0.01$ in $2 \mathrm{D}$ for LD simulations and $\beta=0.81 \pm 0.01$ in $2 \mathrm{D}$ and $\beta=0.91 \pm 0.01$ in $3 \mathrm{D}$ for $\mathrm{MD}$ simulations, as predicted by Chuang et al. [5], where $\beta=2 / \alpha=2 /(1+2 \nu)$ gives 0.80 in $2 \mathrm{D}$ and 0.92 in $3 \mathrm{D}$.

All the above results demonstrate that $\alpha=1+2 \nu$ and $\beta$ $=2 /(1+2 \nu)$ for the range of $N$ studied here. Recently, Wolterink et al. [15] presented different results for unbiased translocation using a 3D lattice model with $\mathrm{MC}$ simulations. According to their scaling argument, $\tau \sim N^{1+2 \nu} \phi\left(b / R_{g}\right)$, where $b$ is the pore width and the scaling function $\phi(x)$ $\sim x^{-0.38 \pm 0.08}$ for $x \rightarrow 0$. This leads to $\tau \sim N^{2.40 \pm 0.08}$ in 3D. For $\tau / N^{1+2 \nu}$ as a function of $N$, as shown in the inset of Fig. 1, we find that the scaling function $\phi\left(b / R_{g}\right)$ does not depend on $N$, in contrast to their claims. Furthermore, they have also computed $\left\langle s^{2}(t)\right\rangle \sim t^{\beta}$, with $\beta=0.81$ in $3 \mathrm{D}$ [16]. In addition, in a more recent paper [17], two of these authors argue that $\beta=(1+\nu) /(1+2 \nu) \approx 0.73$ in 3D for $t<\tau_{R}$ and it crosses over 


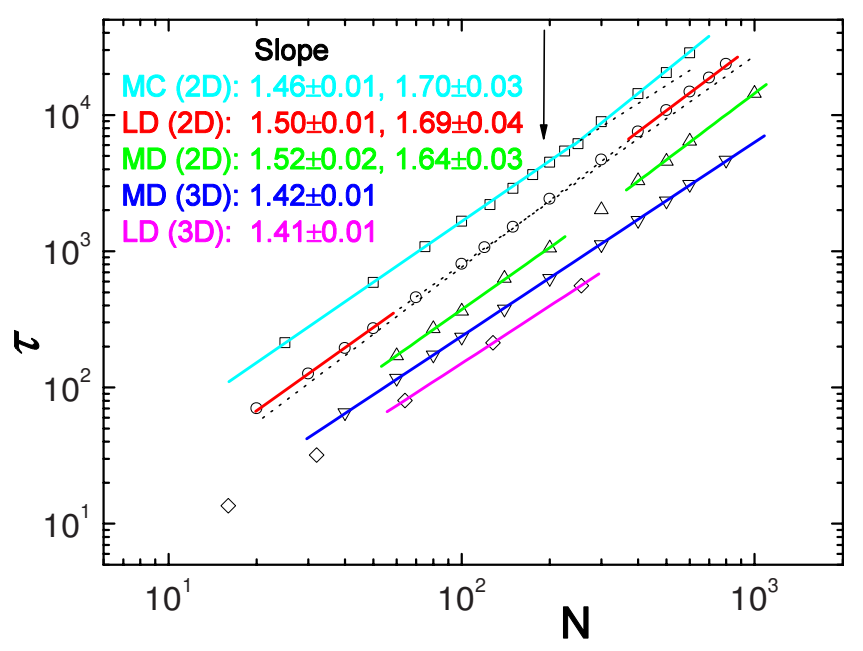

FIG. 3. (Color online) Scaling of translocation times under driving force. The curves have been shifted for clarity. In 2D, the crossover from $\alpha \approx 2 \nu$ to $\alpha \approx 1+\nu$ is observed for all the simulations. In $3 \mathrm{D}$, there is no clear evidence of such a crossover (see text for details). Solid lines indicate fitted data points.

to $\beta=1$ for $t>\tau_{R}$. Correspondingly, they have changed their previous results to $\tau \sim N^{2+\nu}$, where the exponent is 2.75 in $2 \mathrm{D}$ and 2.588 in 3D. In the data shown in Fig. 2, there is no sign of such crossover to $\left\langle s^{2}(t)\right\rangle \sim t$ even at the longest times studied. In fact, $\tau_{R}$ is the relaxation time for the whole chain without confinement. During the translocation process, the chain is always confined by the pore and thus it is impossible for the whole chain to be relaxed even if $t>\tau_{R}$. Therefore, a crossover to the regime where $\beta=1$ cannot possibly exist. Most recently, based on the fractional Fokker-Planck equation, Dubbeldam et al. [19] have argued that $\beta=2 /(2 \nu+2$ $\left.-\gamma_{1}\right)$ and $\tau \sim N^{2 / \beta}=N^{2 \nu+2-\gamma_{1}}$. This gives $\alpha=2.554$ and $\beta$ $=0.78$ in $2 \mathrm{D}$, where $\gamma_{1}=0.945$ in $2 \mathrm{D}$ and $\alpha=2.496$ and $\beta$ $=0.80$ in $3 \mathrm{D}$. However, our results disagree with these claims as well.

Various heuristic scaling arguments for $\tau$ have been presented, e.g., in Refs. [11,12,18,20,21] and will be gauged against our numerical results below. The simple scaling argument presented in Ref. [11] gives $1+\nu$ as the upper bound. Using a more general scaling form with $F R_{g} / k_{B} T$ as the relevant combination [20] gives the result that the exponent is bounded between $2 \nu$ and $1+\nu$, while using $F N / k_{B} T$ gives different results [21]. Reference [18] also suggests $2 \nu$ as a lower bound.

The translocation time as a function of the polymer length is presented in Fig. 3. One of the main features is that a crossover scaling behavior is observed in 2D using different models. For short chains $N \leqslant 200, \alpha=1.46 \pm 0.01$ was found for MC simulations [12] and $\alpha=1.50 \pm 0.01$ for LD simulations [7], and here we find $\alpha=1.52 \pm 0.02$ for MD simulations, all of which are consistent with $\tau \sim N^{2 \nu}$. For longer chains, the exponents cross over to $\alpha=1.70 \pm 0.03$ for $\mathrm{MC}$ simulations [12], $\alpha=1.69 \pm 0.04$ for LD simulations [7], and $\alpha=1.64 \pm 0.03$ for MD simulations, which are slightly below the estimate $\tau \sim N^{1+\nu}$. For simulations in 3D, however, we find no clear evidence of a crossover predicted by the scaling argument for the range of $N$ studied here. For $N=8-32$ the

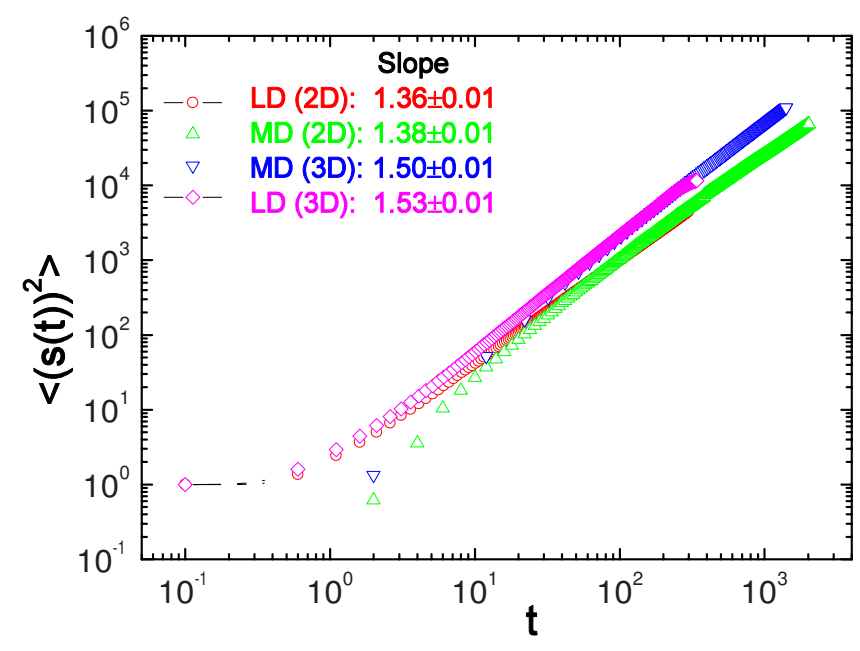

FIG. 4. (Color online) Scaling of the mean-square displacement of the translocation coordinate for driven translocation. The fitting regimes are $10 \leqslant t \leqslant 1500$ ( $2 \mathrm{D} \mathrm{LD}), 60 \leqslant t \leqslant 2020$ ( $2 \mathrm{D} \mathrm{MD}), 40$ $\leqslant t \leqslant 1416$ (3D MD), and $10 \leqslant t \leqslant 340$ (3D LD).

effective $\alpha$ (running slope) is close to $2 \nu$; however, it rapidly increases with $N$, saturating to a value of $\alpha=1.42 \pm 0.01$, which is our best estimate from the new MD data up to $N$ $\leqslant 800$. We note that this value lies between $2 \nu$ and $1+\nu$. Thus, one possible explanation is that the scaling regime $\tau$ $\sim N^{1+\nu}$ in $3 \mathrm{D}$ lies beyond the values of $N$ studied so far. The MD result is fully consistent with LD data in 3D where $\alpha$ $=1.41 \pm 0.01$. As emphasized in the previous works, driven translocation is a highly nonequilibrium process [12] and thus simple scaling arguments may not be accurate. Nonequilibrium effects are expected to be more pronounced in $3 \mathrm{D}$ as compared to the $2 \mathrm{D}$ situation. We indeed find that some aspects of the driven translocation dynamics are sensitive to the physical system parameters, such as polymer-pore interactions [14]. Details of these results will be published elsewhere [28].

In Fig. 4, we show our data for $\left\langle s^{2}(t)\right\rangle \sim t^{\beta}$, where $\beta$ $=1.36 \pm 0.01$ in $2 \mathrm{D}$ and $\beta=1.53 \pm 0.01$ in $3 \mathrm{D}$ for LD simulations with $N=128$ and $\beta=1.38 \pm 0.01$ in $2 \mathrm{D}$ and $\beta$ $=1.50 \pm 0.01$ in $3 \mathrm{D}$ for MD simulations with chain lengths $N=100$ and $N=500$, respectively. These numerical results show that $\alpha \beta=2$ for driven translocation in 2D. However, in 3D $\alpha \beta \approx 2.2$.

Dubbeldam et al. [21] have argued that $\left\langle s^{2}(t)\right\rangle \sim t^{\beta}$, where $\beta=4 /\left(2 \nu+2-\gamma_{1}\right)$. This gives $\beta=1.56$ in $2 \mathrm{D}$ and 1.60 in $3 \mathrm{D}$. They further obtain $\alpha=2 /(\beta / 2)-1=2 \nu+1-\gamma_{1}$, which gives $\alpha=1.55$ in $2 \mathrm{D}$ and $\alpha=1.50$ in 3D. Most recently, Panja et al. [18] have argued that $\beta=2(1+\nu) /(1+2 \nu)$, which is 1.40 in $2 \mathrm{D}$ and 1.46 in 3D. Numerically, they find that $\tau$ $\sim N^{(1+2 \nu) /(1+\nu)}$, which is 1.43 in $2 \mathrm{D}$ and 1.37 in $3 \mathrm{D}$. This result also implies $\alpha \beta=2$. Using the same argument as Storm et al. [23], Panja et al. [18] further claim that the lower bound for $\alpha$ is $\tau \sim N^{2 \nu}$, which gives $\alpha=1.50$ in 2D. Obviously, this contradicts both the prediction $\tau \sim N^{1+\nu}$ in Ref. [11] and the current and previous simulation results $[7,12,13]$. 
To conclude, the dynamics of polymer translocation has been extensively investigated by several independent models in both 2D and 3D to conclusively determine the dynamical scaling exponents. We have focused on the translocation time $\tau$ as a function of the chain length $N$ and the mean-square change of the translocation coordinate $\left\langle s^{2}(t)\right\rangle \sim t^{\beta}$. For unbiased translocation, numerical results are fully consistent with $\alpha=1+2 \nu$ and $\alpha \beta=2$. For driven translocation, numerical results are again consistent with a crossover from $\alpha \approx 2 \nu$ for short chains to $\alpha \approx 1+\nu$ in $2 \mathrm{D}$, where the relation $\alpha \beta=2$ is also valid. In $3 \mathrm{D}, 2 \nu<\alpha=1.42<1+\nu$ and $\alpha \beta \approx 2.2$ for 40 $\leqslant N \leqslant 800$. These results cast serious doubt on the alternate scaling scenarios in Refs. [15-19,21].

This work has been supported by the Academy of Finland through the TransPoly and COMP CoE grants and NSERC of Canada (M.K.). We thank the SharcNet computing facility (www.sharnet.ca) and CSC Ltd. for computer resources.
[1] J. J. Kasianowicz, E. Brandin, D. Branton, and D. W. Deamer, Proc. Natl. Acad. Sci. U.S.A. 93, 13770 (1996).

[2] A. Meller, J. Phys.: Condens. Matter 15, R581 (2003).

[3] W. Sung and P. J. Park, Phys. Rev. Lett. 77, 783 (1996).

[4] M. Muthukumar, J. Chem. Phys. 111, 10371 (1999).

[5] J. Chuang, Y. Kantor, and M. Kardar, Phys. Rev. E 65, 011802 (2001).

[6] K. Luo, T. Ala-Nissila, and S. C. Ying, J. Chem. Phys. 124, 034714 (2006).

[7] I. Huopaniemi, K. F. Luo, T. Ala-Nissila, and S. C. Ying, J. Chem. Phys. 125, 124901 (2006).

[8] I. Huopaniemi, K. F. Luo, T. Ala-Nissila, and S. C. Ying, Phys. Rev. E 75, 061912 (2007).

[9] D. Wei, W. Yang, X. Jin, and Q. Liao, J. Chem. Phys. 126, 204901 (2007).

[10] A. Milchev, K. Binder, and A. Bhattacharya, J. Chem. Phys. 121, 6042 (2004).

[11] Y. Kantor and M. Kardar, Phys. Rev. E 69, 021806 (2004).

[12] K. Luo, I. Huopaniemi, T. Ala-Nissila, and S. C. Ying, J. Chem. Phys. 124, 114704 (2006).

[13] K. Luo, T. Ala-Nissila, S. C. Ying, and A. Bhattacharya, J. Chem. Phys. 126, 145101 (2007).

[14] We find that increasing the pore width by a factor of 2 does not change the $3 \mathrm{D}$ scaling presented here. Interaction effects are discussed in K. Luo, T. Ala-Nissila, S. C. Ying, and A. Bhattacharya, Phys. Rev. Lett. 99, 148102 (2007); 100, 058101 (2008).

[15] J. K. Wolterink, G. T. Barkema, and D. Panja, Phys. Rev. Lett. 96, 208301 (2006).

[16] D. Panja, G. T. Barkema, and R. C. Ball, J. Phys.: Condens.
Matter 19, 432202 (2007).

[17] D. Panja, G. T. Barkema, and R. C. Ball, e-print arXiv:condmat/0610671.

[18] D. Panja, G. T. Barkema, and R. C. Ball, J. Phys.: Condens. Matter 20, 075101 (2008); H. Vocks, D. Panja, G. T. Barkema, and R. C. Ball, ibid. 20, 095224 (2008).

[19] J. L. A. Dubbeldam, A. Milchev, V. G. Rostiashvili, and T. A. Vilgis, Phys. Rev. E 76, 010801(R) (2007).

[20] A. Yu. Grosberg, S. Nechaev, M. Tamm, and O. Vasilyev, Phys. Rev. Lett. 96, 228105 (2006).

[21] J. L. A. Dubbeldam, A. Milchev, V. G. Rostiashvili, and T. A. Vilgis, Europhys. Lett. 79, 18002 (2007).

[22] S. Guillouzic and G. W. Slater, Phys. Lett. A 359, 261 (2006); M. G. Gauthier and G. W. Slater, Eur. Phys. J. E 25, 17 (2008).

[23] A. J. Storm, C. Storm, J. Chen, H. Zandbergen, J.-F. Joanny, and C. Dekker, Nano Lett. 5, 1193 (2005).

[24] H. A. Kramers, Physica (Amsterdam) 7, 284 (1940).

[25] P. G. de Gennes, Scaling Concepts in Polymer Physics (Cornell University Press, Ithaca, NY, 1979); M. Doi and S. F. Edwards, The Theory of Polymer Dynamics (Clarendon, Oxford, 1986).

[26] D. van der Spoel, E. Lindahl, B. Hess, G. Groenhof, A. E. Mark, and H. J. C. Berendsen, J. Comput. Chem. 26, 1701 (2005).

[27] M. Patra, E. Salonen, E. Terama, I. Vattulainen, R. Faller, B. W. Lee, J. Holopainen, and M. Karttunen, Biophys. J. 90 , 1121 (2006).

[28] A. Bhattacharya et al. (unpublished); M. Karttunen et al. (unpublished). 